\title{
Huldigung des Gefühls im 17. Jahrhundert
}

\author{
Kulturlinguistische Analyse schwedischer \\ Gelegenheitskompositionen im Kontext höfischer Zeremonien
}

\author{
Dessislava Stoeva-Holm
}

Online publiziert: 28. März 2019

(C) Der/die Autor(en) 2019

Zusammenfassung Aus einer kulturanalytisch-linguistischen Perspektive wird im Beitrag die Gelegenheitskomposition als eine für das 17. Jahrhundert frequent eingesetzte multikodale Textsorte betrachtet, die mit ihrer Huldigungsfunktion für Zeremonien am Hofe unumgänglich war. Als mehrdimensionale, dichte semantische Geflechte sind diese Kompositionen aber auch stark der Gefühlsvermittlung und -erzeugung verpflichtet, die an Konzepten, Metaphern und Topoi ablesbar sind, d.h. an semantischen Einheiten auch jenseits der Wort- und Satzsemantik. Der Beitrag ist aber nicht nur der Semantik komplexer sprachlicher Zeichen verpflichtet, sondern soll durch Kontextualisierung Aufschluss geben, wie in Zeremonien Sinn geschaffen wurde, indem Emotionen für gesellschaftspolitische Zwecke funktionalisiert wurden.

Schlüsselwörter Gelegenheitskomposition · Emotionen · Erwartungshaltung · Kulturwissenschaftliche Linguistik · Sprachgebrauchsmuster · Dübensammlung · Schwedenkönig Karl XI · Ulrika Eleonora von Dänemark

\section{Tribute to Emotions in the 17th Century}

Cultural-Linguistic Analysis of Swedish Occasional Music in the Context of Ceremonies at the Court

\begin{abstract}
Occasional music was frequently used in the 17th century and indispensable for court ceremonies. From a cultural-analytical and linguistic perspective, the contribution considers occasional music for the purpose of homage as a multi-code type of text. For the analysis of the compositions, concepts, metaphors and topics are taken into account; this refers to semantic units beyond the semantics of words and sentences. However, the focus of the article is not only on the semantics of
\end{abstract}

D. Stoeva-Holm $(\bowtie)$

Institut für moderne Sprachen, Universität Uppsala, Uppsala, Schweden

E-Mail: dessislava.stoeva.holm@moderna.uu.se 
complex linguistic signs, but it is also intended to show by contextualization how meaning was created in ceremonies by functionalizing emotions for socio-political purposes.

Keywords Occasional Music · Emotions · Expectations · Cultural Studies · Patterns in Language Use · The Düben Collection · King of Sweden Karl XI • Ulrika Eleonora of Denmark

\section{Einleitung}

In der Geschichtsschreibung wird das 17. Jahrhundert als Schwedens Großmachtzeit beschrieben. Mit Karl XI. (*1655, †1697) als König wurde nicht nur die Alleinherrschaft des Monarchen etabliert und das schwedische Rechtswesen reformiert, sondern auch die Kriege gegen Dänemark gewonnen. In diesem gesellschaftlichpolitischen Kontext heiratete der Schwedenkönig Karl XI. die dänische Prinzessin Ulrika Eleonora $(* 1656, \uparrow 1693)$. Die Eheschließung wird von den Historikern als politische Handlung zur Befestigung der nachbarlichen friedlichen Koexistenz zwischen Dänemark und Schweden gedeutet, die den Friedensvertrag von 1680 zwischen Schweden und Dänemark besiegeln sollte. Die Hochzeit fand am 6. Mai 1680 auf dem Herrensitz Skottorp, in der Provinz Halland statt und die Braut sollte fast ein halbes Jahr später als Friedensbringerin ihren Einzug in Stockholm halten.

Wie alle anderen gesellschafts-politischen und ökonomischen Erfolge lieferte auch die Hochzeit einen Anlass zum Feiern. So bot sich erneut in der Zeit des Barock die Gelegenheit Schwedens Stellung als Großmacht im Norden und Baltikum deutlich zu demonstrieren und die Befehlsgewalt des Regenten zu markieren. Weder die Wahl des Platzes für die Eheschließung noch die verhältnismäßig begrenzte Anzahl der Hochzeitsgäste waren ein Hindernis dafür, diese Hochzeit gebührend zu feiern. So durfte eine künstlerische Gestaltung der Zeremonie nicht fehlen, denn Kunst stand in Zeiten Karl XI. im Dienste der Perfektionierung einer Festkultur ähnlich wie es am königlichen Hof in Paris oder in Venedig der Fall war (s. Erben 2004, S. 298). Auch wenn die eher anspruchslose Hochzeit im engeren Kreis stattfand, sollten sich mit Hilfe der Kunst die angestrebte Glorifizierung des Herrschers und die Inszenierung seiner Macht vor der zeitgenössischen Öffentlichkeit, vor allem vor ausländischen Potentaten, entfalten. An ökonomischen Ressourcen wurde seitens Karl XI. nicht gespart und die bedeutendsten Künstler, Dichter und Komponisten der Zeit wurden engagiert (vgl. Snickare 1999, S. 8 f.).

Die für die Hochzeit angewandten Inszenierungen der Königsmacht sollten denen anderer Regenten wie z.B. denen von Ludwig XIV. (wie bei Burke 2009 beschrieben) nicht nachstehen. Die Kunst und ihre unterschiedlichen Ausdrucksformen wie Architektur, Malerei, Musik und darstellende Kunst unterlagen dieser Funktionalisierung. Neben diesen waren aber auch insbesondere die Sprache und der Sprachgebrauch in künstlerischen Werken das Instrument, um feierlichen Anlässen die notwendige Gewichtung und den angestrebten, prachtvollen Glanz zu verleihen. Schriftzeugnisse wie Gelegenheitskompositionen (insbesondere Vokalkompositionen mit ihren Texten und Notenblättern), die am schwedischen Hof entstanden, 
geben heute Auskunft darüber, dass vertonte Texte in unterschiedlichen Sprachen den König huldigten und seiner Herrscherlegitimation dienten. Als Zeugnisse ihrer Zeit betrachtet, ist aus diesen Kompositionen aber auch ersichtlich, dass sie im protestantischen Schweden nicht nur zur Lobpreisung des Königs und der Inszenierung seiner Macht benutzt wurden, sondern zu den wenigen Möglichkeiten gehörten, an eine Herrscherethik, d.h. was von einem Regenten erwartet wurde, zu gemahnen und ihre Beachtung - wenn auch indirekt - einzufordern. In zeremoniellen $\mathrm{Zu}$ sammenhängen konnten Gelegenheitskompositionen somit eine weitere politischsoziale aber auch kulturelle Funktion übernehmen, die sich über den Rahmen von Huldigung, Gratulation und Unterhaltung hinaus bewegt. Dass hierbei Emotionen und deren Verbalisierung den thematischen Inhalt von Gelegenheitskompositionen beeinflussen und prägen, soll hier aufgezeigt werden. Unter Emotionen wird »das Insgesamt der kulturellen Vorstellungen und Annahmen, die von einem Kollektiv (einer ganzen Gesellschaft oder von Teilen) mit einer bestimmten Emotion verbunden werden « verstanden (Lenz 2003, S. 256). Die Lesbarkeit dieser kulturellen Vorstellungen und Annahmen zu Emotionen ist, wie zu zeigen wird, an bestimmte Kontexte geknüpft. Der Grundsatz für ein analytisches Vorgehen bei der Analyse von Gelegenheitskompositionen ist deshalb, dass diese multikodalen (verschiedene Zeichenarten enthaltenden) Texte ihre Bedeutung durch Beteiligung unterschiedlicher Zeichensysteme erhalten wie Musik oder auch Stimmführung usw.

»Da alle diese Zeichen gemeinsam Sinn anbieten, da sie alle auf der Textoberfläche und in der Textumgebung etwas zu verstehen geben und wahrgenommen werden sollen, kann man an ihnen nicht vorbeigehen. So kann man nicht den einen Kode, den sprachlichen, aus dem Textkomplex herauslösen und an ihm den Sinn des Ganzen ablesen wollen [...].« (Fix 2008, S. 31, Kursivierung im Original)

\section{Die Gelegenheitskomposition im Kontext der Zeremonie}

Immer wieder wird in der kulturhistorisch ausgerichteten Forschung betont, dass Zeremonien in ihrem kulturellen, sozialen und politischen Zusammenhang zu sehen sind und zeremonielle Akte wie z.B. Huldigungen »streng reguliert« sind und das »elaborierte System sozialer Distinktionen am Hof « zur Schau stellen (Braungart 1996, S. 65). Angefangen bei Edward Gibbon (1776-1788), der die Zeremonie als wichtiges politisches Werkzeug in den Händen der Macht sah, um die Ehrerbietung des Volkes und die Untertänigkeit zu stärken bis zu Jacob Burckhardts (1860) Auffassung von der Zeremonie als hochstehendes Moment im Leben der Bevölkerung in der Renaissancezeit, wo religiöse, moralische und poetische Ideale deutlich Gestalt annehmen und wo Zeremonien einen Übergang des Lebens in die Kunst markieren. Snickare (1999, S. 13) spitzt diese Aussage zu und betrachtet die Zeremonie als ein politisches Ereignis im weiteren Sinne, denn sie sei eine symbolische Handlung, die Aufschluss gibt, wie die Gesellschaft aufgebaut sei und wie sich die Beziehungen zwischen den Ständen gestalten würden. 
Nach Clifford Geertz (1991) verleiht die Zeremonie durch ihre definierte Form dem Geschehen einen Sinn und einen Rahmen. Ihre Aufgabe sei es, die menschliche Erfahrung begreiflich und sinnvoll zu machen. Die Zeremonie erwächst aus dem sozialen Leben der Menschen und ihre Funktion ist es, Sinn und Ordnung aus Unordnung und Chaos zu schaffen. Für ihn ist »die Zeremonie der Ort [...], an dem die Stimmungen und Motivationen, die die religiösen Symbole in den Menschen hervorrufen, und die die allgemeinen Vorstellungen von der Seinsordnung, die sie für die Menschen ausdrücken, zusammentreffen und sich gegenseitig verstärken [...].« Er fährt fort: »Im Ritual sind gelebte und vorgestellte Welt ein und dasselbe, sie sind in einem einzigen System symbolischer Formen verschmolzen.« (Geertz 1991, S. 112f.) Die Zeremonie und ihre Bestandteile werden als ein Ereignis gesehen, das sich an rational handelnde Rezipienten richtet und die in ihrer zeremoniellen Ausstaffierung, der Emblematik und den Allegorien gedeutet werden kann (vgl. Strong 1973; Burke 2009). Zeremonien werden somit hauptsächlich als intellektuell überformte Gestaltung gesehen, um zur Festigung und Legitimierung von herrschenden Strukturen beizutragen. In dramaturgischen Inszenierungen von Feierlichkeiten mit Musik und Gesang kann sich aber eine Wechselwirkung zwischen Zeremonien als kulturellem Muster und Gefühlen als menschlichem Bedürfnis in strikt geregelter Form entfalten. Hierbei bilden Vokalkompositionen durch die Verbindung musikalischer Affekte mit emotionsthematisierenden Texten ein geeignetes Medium diese Wechselwirkung zu konsolidieren, wobei gleichzeitig aus den Versprachlichungen deutlich wird, wie Gefühle inszeniert und konzeptualisiert werden. Durch die Teilnahme an Zeremonien ist der Mensch somit nicht nur wie Victor Turner (1988, S. 81) hervorhebt ein homo performans (ein Mensch, der sich selbst, seine Mitmenschen und die Welt kennenlernt, indem er an Zeremonien teilnimmt und diese betrachtet) und auch nicht nur wie bei Barbara Myerhoff (1978, S. 272) ein homo narrans (ein Mensch, der durch Narration Kohärenz und Ordnung im Chaos schafft und ein soziales und persönliches Ethos formuliert), sondern auch ein homo sentiens - ein Mensch, der zu empfinden und nachempfinden in der Lage ist und dies semiotisch zu kodieren und decodieren versteht.

Gerade im Zeitalter des Barock, in dem es im höchsten Maße galt Gefühle verbal und non-verbal zu inszenieren und zu vermitteln, bildete das Kodieren von Emotionen in Form von Versprachlichung und Vertonung einen zentralen Aspekt im sozialen und kommunikativen Geschehen. In einem zeremoniellen Rahmen eingebettet wird die Affektgestaltung und -vermittlung aber auch zu einem Muster und zur Tradition, wobei Sprache die Rolle übernehmen kann, das Geschehen in der Zeremonie nicht nur mit der Vergangenheit zu verlinken, sondern auch mit der Zukunft und zu einer sinnvollen und geordneten Einheit zu formen. Vokalkompositionen, die zu speziellen Anlässen komponiert wurden und als etablierte Kunstform der Frühen Neuzeit Bestandteil einer Festkultur im weitesten Sinne waren, übernahmen die Funktion der Affektgestaltung und -vermittlung mit Hilfe von Sprache und Musik. 


\section{Emotionen in Gelegenheitskompositionen: Disziplinäre Zugänge ihrer Erfassung}

Gefühle sind als kulturell kodiert zu verstehen, die ihren Sinngehalt erst dann zugeschrieben bekommen, wenn eine Einbettung in komplexere Wissenssysteme geschieht. Diese Wissenssysteme können das kulturelle Wissen über Gefühle und ihren verbalen und non-verbalen Ausdruck beinhalten; diese Wissenssysteme können aber auch die Kenntnis vom Stellenwert der Gefühle in sozialen Praktiken wie Zeremonien enthalten. Wichtig ist dabei, dass sowohl der verbale und non-verbale Ausdrucks, als auch die situative Angemessenheit einer Emotion sowie das Erleben derselben reguliert wird.

Für die Thematisierung von Gefühlen - ein Verfahren, das Strukturen der Huldigung aufheben und trotzdem eine Herrscherethik einfordern kann - können sowohl verbale Mittel wie Metaphern, Allegorien, rhetorische Pathosformeln in den Dienst dieser Aufgabe gestellt werden, als auch non-verbale wie die musikalische Gestaltung. Dabei spielt das Wissen, wie musikalische Affekte erzeugt werden, als auch die situative Einbettung einer Komposition in eine Zeremonie eine gewichtige Rolle, um Gefühle zu inszenieren.

\subsection{Affektenlehre in den Musikwissenschaften}

Die Aufgabe der Musik, ihre Zweckbestimmung, im 17. Jahrhundert kann als zweigeteilt aufgefasst werden. Eine Aufgabe war die Funktionsästhetik, der zufolge das einzelne Werk eine Gattungsnorm vertritt, um als decorum eine soziale Funktion zu erfüllen. Die andere Aufgabe war die Repräsentationsästhetik, der zufolge die Aufgabe der Musik es war, einen Affekt hervorzubringen oder eine allegorische oder symbolische Bedeutung zu schaffen. (Berglund 2002, S. 17) Für die Musik allgemein waren somit Affektgestaltung und -vermittlung innerhalb der Affektenlehre in der Frühen Neuzeit von grundlegender theoretischer und empirischer Wichtigkeit. Jukka Saralja (2001, S. 17) zufolge, war dies »not just a theory among others; it was the theory of music and its characteristics «.

Kunst wurde als Wissen, das auf Kenntnis bestimmter Regeln fußte, gesehen und Musiktheoretiker aus dem 17. und 18. Jahrhundert diskutierten - in diesem Zusammenhang betrachtet - eingehend Fragen, wann, warum, auf welcher Art und Weise Affekte musikalisch repräsentiert werden. Da davon ausgegangen wurde, dass Sprache und Musiksprache (Musica Poetica) eine gemeinsame Grundlage haben, führte dies dazu, die Affektenlehre der Barockzeit eng mit der Affektenlehre der Rhetorik zu verknüpfen.

Johann Mattheson gibt im Der Vollkommene Capellmeister (1739), einige wenige Kommentare zu den Affekten, die an dieser Stelle genannt werden sollten: Freude »wird am besten durch weite und erweiterte Intervalle ausgedrückt« (S. 16, §56) und Stolz - Hochmut - Hoffart »werden am besten durch prächtig klingende Figuren, die eine besondere Ernsthaftigkeit und hochtrabende Bewegung erfordern, ausgedrückt, niemals aber viel Flüchtiges und Fallendes zulassen, sondern immer nur steigen wollen« (S. 18, §72) 
In heutigen Arbeiten von Musiktheoretikern werden weiterhin Versuche unternommen, Affekte zu bestimmen (vgl. Schildt 2014). Auch diese Arbeiten sind auf die Fragen, wann und warum Affekte repräsentiert sind, ausgerichtet, doch sei es schwieriger zu ersehen, welche technisch-musikalischen Mittel einem Komponisten zur Verfügung standen, um in allerbester Weise diese Affekte zu vermitteln. Maria Schildt (2007, S. 31) zufolge gibt es nur wenige und widersprüchliche Studien dazu. Methodisch bietet sich der Vergleich als Herangehensweise an, wie er z. B. von Lars Berglund (2002), der Gelegenheitskompositionen von Christian Geist mit Musik aus derselben Zeit und für ähnliche Anlässe verglichen hat, durchgeführt worden ist. In seiner musikwissenschaftlichen Aufarbeitung konnte er folgende Gemeinsamkeiten zwischen Kompositionen feststellen, die anlässlich der Volljährigkeit von Karl XI. komponiert wurden:

»tonarten är c-dur (eller formulerad som jonisk på c)

inslag av tuttisektioner i tretakt med växlande betoningsmönster och hemioler (courantmönster)

inslag av upprepade sextondelsfigurer, vilka ger en >krigisk affekt< (jfr Monteverdi)

harmoniskt och satstekniskt förhållandevis enkla modeller och rätlinjig mall inslag av treklangsfigurer $\ll^{1}$ (Berglund 2002, S. 220)

Textinhalte und verbale Gefühlskodierungen werden in diesen Arbeiten nur am Rande erwähnt, wenn überhaupt. In Bezug auf den Text der Gelegenheitskompositionen verweisen Musikwissenschaftler auf die Rhetorik und übergehen z. B. neuere linguistische Forschung zu Affekten und Emotionen. Der Zusammenhang zwischen den zwei semiotischen Systemen Text und Musik erscheint somit in der musiktheoretischen Forschung unterbelichtet, wogegen man das Augenmerk eher auf den Anlass der Komposition richtet. Weil im Folgenden von einem determinierenden Zusammenhang von Sprache und Emotionskultur ausgegangen wird, soll hier insbesondere auf das Zusammenspiel von Sprache und Emotion in einem zeremoniellen Rahmen eingegangen werden.

\subsection{Emotionen und Linguistik}

Nach Harré (1986) gibt es verschiedene Bedingungen, die den Gebrauch von sprachlichen Gefühlsausdrücken veranlassen, die jedoch nicht alle erfüllt sein müssen:

- physische emotionsspezifische Empfindung;

- externe Gegenstände oder Situationskonstellationen;

\footnotetext{
1 »Die Tonart ist C-Dur (oder ionisch auf C formuliert);

Elemente von Tutti-Passagen im Dreiertakt mit unterschiedlichen Betonungsmustern aus Hemiolen (Courant-Mustern);

Elemente von sich wiederholender Sechzehntel-Figuren, die einen >kriegerischen Affekt< ergeben (vgl. Monteverdi);

harmonisch und satztechnisch relativ einfache Modelle und geradlinige Muster;

Elemente von Drei-Klang-Figuren« [Übers. D.S.-H.].
} 
- Moralordnungen, die sich inhaltlich aufschlüsseln lassen, z. B. in kulturell übliche Verpflichtungen, Regeln, Zwänge, Bewertungskonventionen, Gebote.

Ich gehe davon aus, dass Moralordnungen in einer Gesellschaft und spezifische Sprachgebräuche oft die ausschlaggebenden Kriterien dafür sind, wie und welche emotionale Qualität Zeremonien und künstlerischen Darbietungen zugeteilt werden können. Insbesondere in der Frühen Neuzeit, wo für Zeremonien eine besondere Vorliebe und ein besonderes Verständnis vorlag, konnte das Zeremoniell das Verhalten bis ins Körperliche hinein regulieren. Streng regulierte Huldigungsakte demonstrieren das elaborierte System sozialer Distinktionen am Hof und schlossen ungeregeltes Verhalten weitgehend aus (vgl. Braungart 1996, S. 64f.). Die Festkultur in der Frühen Neuzeit ist der ästhetische Ausdruck eines in dieser Kultur eingebundenen Menschen.

Um emotionale Gehalte in Gelegenheitskompositionen aus kulturlinguistischer Sicht analysieren zu können, kann auf einen linguistischen und kodebasierten Ansatz zurückgegriffen werden, wie ihn Schwarz-Friesel (2007, 2017) für Analysen literarischer Texte, Jahr (2000) für Analysen von Sachtexten im Anschluss an psychologische Emotionsforschung entwickelt hat oder Winko (2003) für literaturwissenschaftliche Analysen von Lyrik im Anschluss an soziologische Emotionstheorien. ${ }^{2}$ Diesem Ansatz zufolge werden Emotionsdarstellungen auf der Textebene untersucht und aus der Art und Weise der Emotionskodierung bzw. der sprachlichen Strukturen Hypothesen des emotionalen Wirkungspotentials abgeleitet. Dieser kodebasierte Ansatz fußt auf folgenden Grundannahmen, die hier unter Bezug auf Winko (2003) und Schwarz-Friesel (2017) aufgeführt werden:

- Textproduzent und -rezipient teilen ein gemeinsames, konventionalisiertes Wissen über Emotionen und prototypisch emotionale Handlungen und Situationen.

- Einheiten und Strukturen, die Emotivität kodieren, lassen sich auf allen sprachlichen Ebenen identifizieren und mittels textlinguistischer Analysekriterien präzise beschreiben und erklären.

- Das Emotionspotential eines Textes konstituiert sich als die Menge aller intersubjektiv erfass- und darstellbaren textinternen Elemente und Informationsstrukturen, die Gefühle abbilden oder ausdrücken sowie Evaluationen vermitteln.

- Das tatsächliche Wirkungspotential der Texte ist nur unter Berücksichtigung textexterner Faktoren zu bestimmen.

Ausgehend von diesem kodebasierten Ansatz zur Darstellung aber auch zur Identifikation von Emotionen scheinen kontextualisierende Analyseverfahren nicht nur möglich, sondern geradezu auf der Hand liegend, da hier eine Verknüpfung von

\footnotetext{
2 Winko (2003) unterscheidet produktions-, rezeptions-, text- und kontextbezogene Ansätze und geht in dem Abschnitt »Rezeptionsbezogene Ansätze« auf die Affektenlehre der Rhetorik ein. Sie bemerkt mit Recht, dass in der gegenwärtigen Rhetorikforschung lediglich eine historisch-rekonstruierende Untersuchungsperspektive dominiert. Für eine kulturanalytische Sprach- und Emotionsforschung könnte hingegen eine systematische Verbindung älterer Einsichten der Rhetoriktheorie mit den Kognitionswissenschaften von erheblichem Gewinn sein, denn diese Verbindung könnte eine Vielzahl der Bestandteile emotionaler Kommunikation in den Blick bekommen - so wie eingesetzte Emotionalisierungstechniken und deren potenzielle und reale Effekte beim Rezipienten.
} 
Textwelt einerseits und Zeremonie andererseits geschehen kann. Dabei kann angenommen werden, dass bestimmte soziale Schichten in der Frühen Neuzeit bestimmtes Wissen über emotionale Muster teilen, d.h., sie verfügen über ein Regelwissen, wie in einer Situation zu fühlen ist, in welchem Umfang Gefühle vorzeigbar und welche Ausdrucksmittel zur Wiedergabe von Gefühlen zu verwenden sind. ${ }^{3}$

Der kodebasierte Ansatz kann verdeutlichen, wie Gefühle in zeremoniellem Huldigungszusammenhang durch sprachliche Einheiten und Strukturen für die Anwesenden inszeniert werden, d.h., welche Verfahren zur Versprachlichung von Gefühlen benutzt werden. Von Inszenierung der Gefühle zu sprechen bedeutet, dass es sich nicht um sprachliche Manifestationen des inneren Zustandes des Sängers oder des Komponisten handelt ${ }^{4}$, sondern um sprachliche Formulierungen kulturell geprägter Muster. Hierbei werden sowohl Konzeptualisierungen als auch Symbolsysteme und somit textspezifische Inszenierungen von Gefühlen offengelegt. Durch die Betrachtung sprachlicher Einheiten auf der lexikalisch-syntaktischen Ebene wird ersichtlich, wie Emotionen im lyrischen Text inszeniert werden und auch wie diese Einheiten durch ihr mehrmaliges Vorkommen, durch Rekurrenz den Text musterhaft formen. Da Vergleiche und Metaphern für den Barock stilbildend sind, können diese insbesondere im Hinblick auf Konzeptualisierungen befragt werden.

Für die sprachliche Darstellung von Gefühlen sind aus der Forschungsliteratur zusätzliche Spezifikationen zu entnehmen, die hier subsummiert angeführt werden. So können nach Schwarz-Friesel (2007, S. 212), Jahr (2000, S. 86 ff.) und Winko (2003, S. 111) zwei grundlegende Kategorien unterschieden werden: die Thematisierung und die Präsentation von Gefühlen, die entweder abhängig oder unabhängig voneinander benutzt werden und die sich in ihrer Funktion entweder gegenseitig betonen oder widersprechen können.

Bei der Thematisierung von Gefühlen in Gelegenheitskompositionen geht es um deren Nennung, der expliziten Referenz oder deren Umschreibung, d.h., was über Gefühle im jeweiligen Text gesagt wird. Das können Definitionen einzelner Gefühle, deren Bewertung und angemessene Ausdrucksweise sein. Thematisierung meint aber auch, mit welchen Begriffen oder Bildern auf Gefühle Bezug genommen wird. Die Analyse der Thematisierung von Gefühlen kann so zweierlei aufzeigen: zum einen deren Funktionalisierung im Einzeltext und zum anderen die Konzeptualisierung und die Rolle von Gefühlen zu einem bestimmten Zeitpunkt oder in einer bestimmten Sprechergemeinschaft (vgl. Stoeva-Holm 2015).

Die Präsentation von Gefühlen in Texten unterscheidet sich von der Thematisierung insofern, als es hier nicht um auf Gefühle bezogene Propositionen geht, sondern um die Vermittlung von Gefühlen beispielsweise wie es bei thematischen Beschreibungen einer Landschaft, einer Situation oder einer Figur in Lyrik oder

\footnotetext{
3 Ekman und von Friesen (1975) nannten diese Regeln feeling rules und display rules.

${ }^{4}$ Dass sich die Ansichten über den Echtheitscharakter von Gefühlen unterscheiden können, darüber gibt die Arbeit von Gerhard Vowinckel (1989, S. 363) Aufschluss. Anhand seiner Analysen von Anstands- und Tugendbüchern aus verschiedenen Jahrhunderten stellt er fest, dass Tugendlehrern im Mittelalter zufolge der freundliche Ausdruck ohne freundliche Gesinnung abwegig war, wogegen von politischen Klugheitslehrern das >Stellen< und >Verstellen< im 17. Jahrhundert zur Kunst der Könige erhoben wurde, um Ende des 18. Jahrhunderts von bürgerlichen Tugendlehrern angeprangert zu werden, die keine Diskrepanz zwischen innerer und ausgedrückter Gesinnung zulassen wollten.
} 
Prosaskizzen sein kann (vgl. Winko 2003, S. 116; Hillebrandt 2011, S. 78-87). Hier spielen vor allem implizite sprachliche Mittel und Strukturen eine Rolle als auch die musikalische Gestaltung. Die Präsentation von Gefühlen kann somit auf sehr vielfältige Weise erfolgen. Prinzipiell sind Mischformen von Thematisierung und Präsentation am häufigsten, wo die präsentierten Emotionen die thematisierten unterstützen, verstärken, zu ihnen im Spannungsverhältnis treten oder sie sogar negieren können.

\section{Die Gelegenheitskomposition als Huldigung und Anweisung im Barock}

Dass Musiker und Hofmusiker beauftragt wurden, zu besonderen Anlässen Kompositionen zu kreieren, deutet darauf hin, dass Gelegenheitskompositionen zu den festen Bestandteilen von Zeremonien im schwedischen Königshaus gehörten. Gelegenheitskompositionen erfreuten sich großer Beliebtheit am Hofe und in adligen Kreisen und höheren Ständen. Sammlungen in Schweden, die Musikalienproduktionen aus dem 17. und 18. Jahrhundert enthalten, wie z. B. die sogenannte Dübensammlung, lässt die große Bedeutung von Gelegenheitskompositionen erkennen. Die Dübensammlung ist ein Archiv mit Vokal- und Instrumentalmusik und gilt in der Musikwelt als einzigartige Barockmusiksammlung, da sie systematisch angelegt worden ist und lückenlos alle Werke in einer Zeitspanne von 80 Jahren enthält, die am schwedischen Hofe während der Herrschaft von vier Regenten entstanden ist: von Königin Christina (1644-1654), über Karl X. Gustav (1654-1660) und Karl XI. (1660 / 1672-1697) bis zu Karl XII. (1698-1718). Sie besteht aus 20000 Notenblättern und etwa 2400 Vokal- und Instrumentalwerken von mehr als 300 Komponisten aus Deutschland, Italien, Frankreich, Polen, England, den baltischen Ländern und Schweden, enthält aber auch eine große Anzahl anonymer Werke. (https://www2. musik.uu.se/duben/DubenCollectionInfo.php)

Dass die Gelegenheitswerke aus dieser Sammlung nur teilweise erforscht sind, liegt sowohl an der bis vor einigen Jahren schwierigen Zugänglichkeit der Musikalien aber auch an Vorbehalten seitens der Forschungsgemeinschaft zu deren Untersuchungswert. Von vielen Musikwissenschaftlern werden Gelegenheitskompositionen oft als handwerklich sauber, aber doch simpel in ihrer Fraktur beurteilt. Die enge Situationsbindung und die weitgehende Standardisierung in der sprachlich-musikalischen Ausgestaltung sind weitere Argumente, die angeführt werden. ${ }^{5}$ Dennoch kann nicht geleugnet werden, dass die große Menge an Gelegenheitskompositionen einen gewichtigen Teil der Hofkultur darstellt, die vor allem unter kulturanalytischem Aspekt ein ergiebiges Forschungsfeld bietet.

\footnotetext{
5 Gelegenheitskompositionen anlässlich des Todes und im Zusammenhang mit Begräbnissen aufgeführt, waren kaum der Ort für Innovationen in kompositorischer Hinsicht und auch kein Ort für Experimente. Deshalb waren die entsprechenden Werke für eine als Kompositionsgeschichte verstandene Musikhistoriographie von geringem Interesse.
} 
Im Folgenden betrachte ich Gelegenheitskompositionen mit dem Text als Ausgangspunkt ${ }^{6}$. Die Texte können allgemeinen Charakter aufweisen, Bibeltexte oder kirchliche Texte allgemein sein, die zu einem bestimmten Zeitpunkt eine Vertonung erhalten haben. Auch wenn die künstlerische Qualität der Gelegenheitskompositionen infrage gestellt werden könnte, so liegt deren Bedeutung in ihrer Einbindung in Rituale und Zeremonien. Gerade in der Situation der Aufführung, der Performanz, entfaltet sich deren Potential. Die enge Anbindung an den situativen Kontext und die Performanz bestimmt die rhetorische Aufgabe der Gelegenheitskompositionen des delectare und movere, die sowohl durch die konkrete Sprachwahl als auch durch die Textinhalte getragen werden sollten. Aus einer kulturanalytischen Perspektive sind folgende Aspekte besonders ausschlaggebend für die Zuwendung zu Gelegenheitskompositionen:

1. Die Gesangstechnik im Barock verändert sich zugunsten einer Rezitationstechnik (vgl. Fischer 1998, S. 29, bes. Fn. 56). Diese rezitative Gesangsart erleichtert es, das gesungene Wort deutlicher akustisch zu erfassen. Somit sind nun auch Voraussetzungen vorhanden, die Texte leichter inhaltlich zu rezipieren, was die Verbreitung von sprachlichen Mustern, Schablonen und Konzepten fördert.

2. Gelegenheitskompositionen im Kontext von Zeremonien ermöglichen einen Zugang zu moralischen Urteilen einer Gesellschaft, die sich im Sprachgebrauch wiederfinden. Emotionen und dazugehörende Emotionsausdrücke bilden dabei die Plattform, auf der die Werturteile transportiert werden.

Wie in Gelegenheitskompositionen Herrscherlegitimierung verbal zum Tragen kommt, aber auch wie Erwartungshaltungen in ihnen an Herrscher herangetragen werden, ist Gegenstand der folgenden Ausführungen. Es geht hierbei um Erwartungshaltungen, die an die Braut Ulrika Eleonora von Dänemark herangetragen wurden. Diese Erwartungshaltungen galt es, an sie nicht nur heranzutragen, sondern durch das Fest und seiner Funktion auch öffentlich zur Schau zu stellen, was mittels des Mediums der Gelegenheitskomposition und der in ihr verankerten Inhalte vollzogen werden konnte.

\section{Hochzeitskompositionen in der Dübensammlung}

Anlässlich der Eheschließung zwischen dem schwedischen König Karl XI. und Ulrika Eleonora von Dänemark wurden nachweislich fünf Gelegenheitswerke in den Sprachen Deutsch, Schwedisch und Latein komponiert, die alle in der Dübensammlung überliefert sind. Folgende Werke wurden während der Hochzeit von Karl XI. und Ulrika Eleonora aufgeführt:

Dietrich Buxtehude: Klinget für Freuden

Gustav Düben: Alles Leben dieser Erden

Gustav Düben: War wälkommen min bästa Wänn ${ }^{7}$ (schw.)

\footnotetext{
${ }^{6}$ Auch in musikwissenschaftlichen Studien zu Gelegenheitskompositionen werden diese Werke als »Texte« aufgefasst (vgl. Schildt 2007, 10).

7 dt. Sei willkommen getreuer Freund.
} 


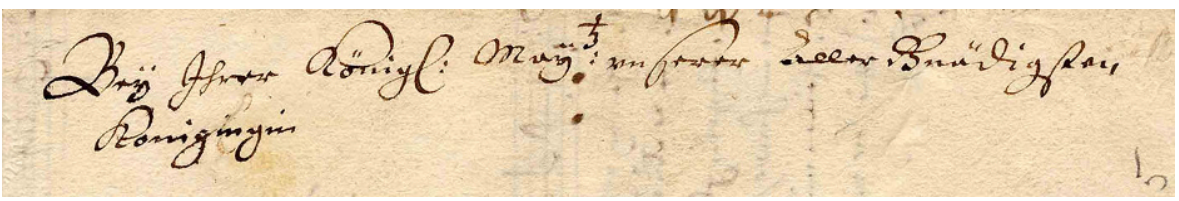

Abb. 1 Zueignung der Komposition von Gustav Düben Alles Leben dieser Erden (1680) (https:// www2.musik.uu.se/duben/browsePart.php?Select_Part=03\&Select_Dnr=689\&command=restart, $\quad$ ges. 8.12.2018).

Christian Geist: Beati omnes qui timent Dominum ${ }^{8}$ (lat.)

Christian Geist: Sij huru godt och lustight är thet ${ }^{9}$ (schw.)

Buxtehudes Klinget für Freuden und Dübens War wälkommen min bästa Wänn waren als Huldigungskompositionen für Karl XI. bestimmt wie auch Geists Kompositionen. Dübens Alles Leben dieser Erden ${ }^{10}$ war der zukünftigen Regentin Ulrika Eleonora zugeeignet ${ }^{11}$ (s. Abb. $1^{12}$ ). Die Zueignung war eine etablierte Form der Huldigung und ist nicht selten in Gedichtbänden aus dem Barock zu finden. Sie bezeugt ein halb höfliches, halb vertrauliches Komplementieren (vgl. Herzog 1979, S. 22).

Diese Hochzeitskomposition ist auch die einzige, die neben der Zueignung für Ulrika Eleonora auch eine Spruchdichtung - auf dem Notenblatt für die Bassstimme - aufweist (s. Abb. $2^{13}$ ), die sich in Aufbau und Inhalt eng an den Text Air de Ballet De M. Batiste von Molière aus dem Jahre 1668 anlehnt $^{14}$.

\footnotetext{
8 dt. Seelig sind alle, die den Herren fürchten.

9 dt. Schau wie schön und lustig es ist.

10 Das ausgewählte Musikstück liegt nur als Handschrift vor und es handelt sich um ein Werk, bei dem Text und Musik gleichzeitig entstanden sind. Dies ist insofern von Bedeutung, da Texte zu schon bereits vorhandener Musik in der Dübensammlung zu finden sind.
}

11 Dem Musikwissenschaftler Tobias Norlind zufolge ist Gustav Dübens Arie Alles Leben dieser Erden der Königin »zugeeignet« (1900, S. 181). Diese Auffassung teilt auch Schildt (2007, S. 42).

12 Beÿ Ihrer Königl: Maÿ:tz vnserer aller Gnädigsten Konigingin.

13 En vain à l'amour

vous estes rebelle.

Dieu quelque jour

vous fera bien voir

jusques ou va son pouvoir

vos attraits n'ont pas esté faicet

Pour vous rendre l'esprit Cruelle

Mais sçachez la Belle

que Malgré la rigueur

l'on peut gaigner un Coeur

qui ne se rends jamais fidelle

que par la douceur.

14 Den Hinweis auf Molières Text, verzeichnet in Alphonse Lemerres Poésies diverses attribuées à Molière ou pouvant lui être attribuées (1869, S. 112) verdanke ich Sylviane Robardey-Eppstein. Molières Text sollte als Gesang im Zusammenhang mit einem Ballett vorgetragen werden. Es ist auch nicht ganz auszuschließen, dass Dübens Vokalkomposition mit einer Ballettdarbietung einherging. 
Abb. 2 Vers in der Komposition von Gustav Düben Alles Leben dieser Erden (1680) (https://www2.musik. uu.se/duben/browsePart.php? Select_Part $=03 \&$ Select_ Dnr $=689 \&$ commaco $=$ restart, ges. 8.12.2018)

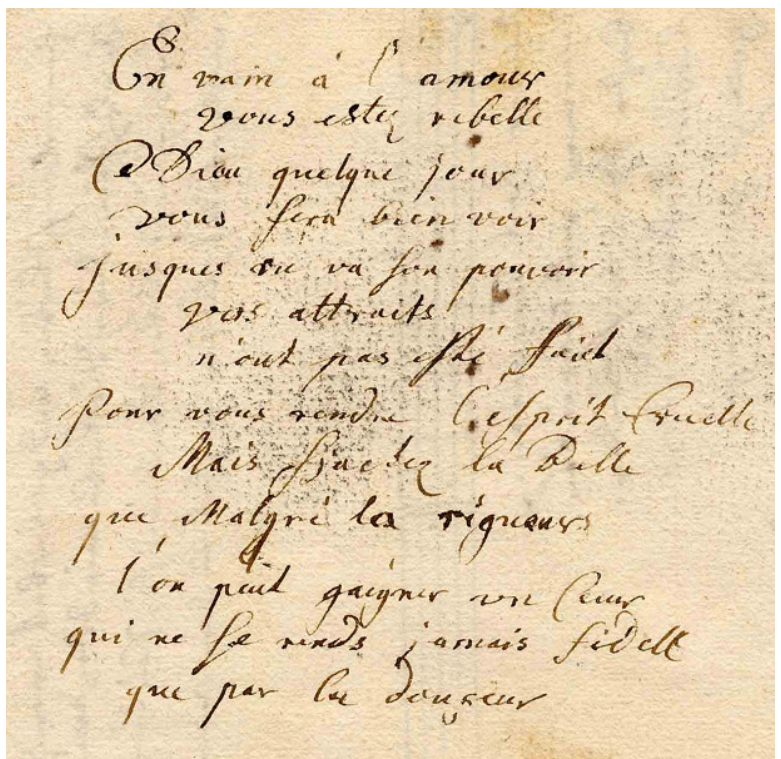

Im Folgenden soll die Komposition Alles Leben dieser Erden ${ }^{15}$ näher betrachtet werden (s. Abb. 3), da zu erwarten ist, dass hier wie in Lobschriften allgemein geltend war, inhaltlich die Königin gepriesen wird und auch Spuren einer Herrscherethik und somit, was von Ulrika Eleonora als Königin erwartet wurde, in dem Text vorzufinden sind. Dies impliziert, dass die künstlerische Ausformung des Textes gattungsspezifisch bestimmten Gestaltungsmustern folgt, dass der Inhalt gängige und zeittypische Vorstellungen von der Rolle der Königin in der Gesellschaft spiegelt und dass konkrete Anforderungen adressaten- und situationsgerecht einen sprachlich angemessenen und korrekten Duktus aufweisen.

Doch wie ist ein Text zu gestalten, in dem eine Regentin gewürdigt werden soll, die auf Grund ihres jungen Alters nur über eine kurze Biographie verfügte, deshalb kaum im gesellschaftspolitischen Rampenlicht stehen konnte und weitgehend unbekannt und die zudem die Schwester des dänischen Königs war, gegen den sich der Bräutigam Karl XI. kürzlich noch im Krieg befand? Wie und in welchem Kontext können des Weiteren Forderungen an Ulrika Eleonora gestellt werden?

Diese Herausforderungen wurden vom Hofmusiker Düben m. E. durch Verschiebung des thematischen Fokus überbrückt, indem er das Gefühl der Liebe als himmlisches, von Gott gegebenes Gefühl inszeniert und als Anlass für die Eheschließung durchscheinen lässt. Insofern ergeben sich Fragen zum Text der Arie, d.h., welche sprachlichen Mittel und Muster hier zu sichten sind, nicht nur in Bezug auf Huldigungszwecke oder für das Einfordern (wenn auch indirekt) einer Herrscherethik, sondern auch in Bezug auf die Kontextualisierung der Heirat als eine Heirat aus Lie-

15 In ihrer musikwissenschaftlichen Untersuchung ist Schildt (2007, S. 42f.) der Ansicht, dass ein anderer Text vorlag, von der man (außer den Titel) den Wortlaut nicht kennt. Die Hochzeitskomposition liegt auch in einer gekürzten Version vor und weist auf Varianten in den musikalischen Besetzungen hin. 


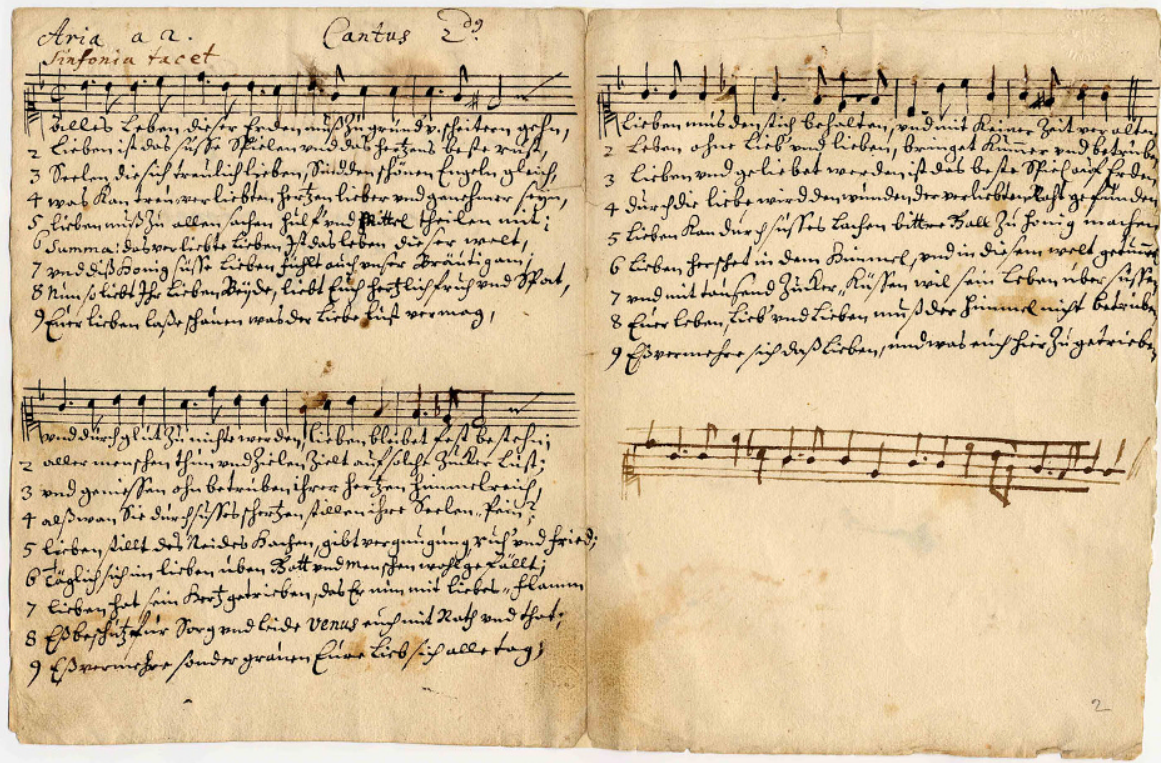

Abb. 3 Notenblatt von Gustav Düben: Sinfonia. Aria. Alles Leben dieser Erden (1680) (http://www2. musik.uu.se/duben/images/vmhs019,001_p02_01v.jpg, ges. 8.12.2018)

be. In den Fokus gelangen somit die Emotionsthematisierungen und -präsentationen in Form von Topoi und sprachlichen Zeichen auf lexikalisch-syntaktischer Ebene und darüber hinaus und somit komplexer Zeichensysteme, deren Gebrauch nicht nur Ausdruck für deren Akzeptanz ist, sondern auch Ausdruck für deren Tradierung durch eine (mehrmalige) Aufführung ${ }^{16}$ ist. Akzeptanz und Tradierung können ein Hinweis auf Modifikationen und Veränderungen von Denkweisen und Konzepten zu Emotionen und deren Thematisierung liefern, d.h., nicht nur wann, wie und wie viel $\mathrm{zu}$ fühlen ist, sondern auch wie über Emotionen zu sprechen ist. Dies ist insofern relevant, als dass sich kulturelle Praktiken am Hof, wie hier die Benutzung von Gelegenheitskompositionen zu besonderen Anlässen oder auch spezifische Formulierungsmuster allmählich auch in niedrigeren Ständen etablieren und somit auch die damit verbundenen Denkweisen und Konzepte. Der Fokus auf diese Gelegenheitskomposition für das Königspaar Karl XI. und Ulrika Eleonora von Dänemark wirft sowohl philologische als auch kulturanalytische Fragen auf, die anhand von Sprachanalysen deutlich machen sollen:

- welchen Aufbau die Gelegenheitskomposition aufweist,

- welche Stilmittel der Affektgestaltung vorzufinden sind,

- mit welchen Topoi wird die Erwartungshaltung an Ulrika Eleonora im Kontext der Zeremonie herangetragen wird, mit anderen Worten, was sagbar ist und wie es gesagt wird.

16 Schildt (2007, S. 43) ist der Auffassung, dass Dübens Arie mehrmals aufgeführt wurde und zwar im Sommer und Herbst des Jahres 1680, während Ulrika Eleonoras Aufenthalten in den Sommerresidenzen der Königinwitwe Hedvig Eleonora. 


\section{Huldigung des Gefühls der Liebe}

Der Sinn der Komposition Alles Leben dieser Erden (1680) wird vorrangig durch die Einbettung in die Zeremonie geschaffen und entfaltet sich durch den Text auf Grund der Textsemantik und dem vorhandenen Referenzpotential. Auf einem gefühlsgeladenen Hintergrund werden in dieser Komposition die Erwartungshaltungen nicht nur an Ulrika Eleonora, sondern auch an Karl XI. formuliert und durch die Aufführung vor einem ausgewählten Publikum öffentlich dargeboten. Indem dies auf Deutsch geschah, geschah es u. a. auch in der Muttersprache der zukünftigen Regentin und in der Sprache, die im Alltag sowohl am dänischen Hof als auch am schwedischen Hof gesprochen wurde. Die Sprachwahl signalisierte nicht nur, wie wichtig es war, dass der Prinzessin aus Dänemark die Botschaft in ihrer Muttersprache dargeboten wurde, sondern auch dass Deutsch einen besonderen Platz für öffentliche Inszenierungen von Gefühlen am schwedischen Königshaus im Zusammenhang von Zeremonien hatte. Auf Deutsch sollten die gesellschaftlichen Erwartungshaltungen in angemessener Art und Weise - Ulrika Eleonora plausibel gemacht und emotional nähergebracht werden als auch eingefordert werden, was in Form von Präsentation und Thematisierung des Gefühls der Liebe geschieht.

1. Alles Leben dieser Erden muß zu Grund wnd scheitern gehn, wnd durch Glut zu nichte werden, lieben bleibet fest bestehn; Lieben muß den Stich behalten wnd mit keiner Zeit weralten.

2. Lieben ist das süsse spielen wnd das Hertzens beste Ruht, Aller Menschen thun wnd Zielen zielt auf solche Zucker Lust; Leben ohne Lieb' wnd Lieben, bringet Kümmer wnd Betruben.

3. Seelen, die sich treulich lieben, sind den schönen Engeln gleich, wnd geniessen ohn Betruben ihrer Hertzen Himmelreich,

Lieben wnd geliebet werden ist das beste Spiel auff Erden.

4. Waß kann treurer liebten Hertzen lieber wnd genehmer seijn, alß wann Sie durch susses Scherzten stillen ihre Seelen Pein?

Durch dir liebe wird den wunden der werliebten Raht gefunden.

5. Lieben muß zu allen Sachen Hulff' wnd Mittel Theilen mit;

Lieben stillt des Neides Hassen, gibt wergnugung, Ruh' wnd Fried;

Lieben kan durch susses lachen bittre Gall zu Honig machen.

6. Summa: das werliebte Lieben ist das leben dieser Welt, täglich sich im Lieben uben Gott wnd Menschen wohlgefält; Lieben herschet in dem Himmel, wnd in diesem Welt getummel.

7. Wnd diß Honig susse Lieben fuhlt auch wnser Bräutigam; Lieben hat sein Hertz getrieben, daß Er nun mit liebes Flamm wnd mit tausend Zucker-Kussen wil sein Leben uber sussen.

8. Nun so liebt Ihr Lieben Beijde, liebt Euch hertzlich fruh wnd Spat, Eß beschütz fur Sorg wnd Leide Wenuß euch mit Rath wnd that; Euer Leben, Lieb' wnd Leben muß der Himmel nicht betruben.

9. Euer Lieben laße schauen was der Liebe Lust wermag, Eß wermehre sonder grauen Eure Lieb sich alle Tag; 
Indem das Gefühl der Liebe während der Hochzeitsfeier als Schwerpunkt inszeniert wird, wird die Beziehung zwischen Braut und Bräutigam als Verbindung aus Liebe bestimmt, wird das Liebesgefühl nicht nur im interpersonellen, sondern auch im politischen Kontext, als bedeutsam und wirksam gestaltet, und es wird der Versuch unternommen etwaige Konflikte oder Krisen zwischen den schwedischen und dänischen Königshäusern mit Hilfe der Präsentation und der Thematisierung des Liebesgefühls zu entschärfen.

\subsection{Präsentation der Emotionen}

Texte in Gelegenheitskompositionen im Barock begegnen in starker musikalischer und textueller Vorgeprägtheit, die jedoch nicht mit inhaltsloser Formelhaftigkeit gleichzusetzen ist. Für die Präsentation von Emotionen dient die deutliche Adressierung des Brautpaares (Strophe 9), des konkreten Anlasses zur Feier und somit außersprachliche Sachverhalte, auf die Bezug genommen wird (Strophe 7). Der Aufbau der Komposition folgt der beliebten Dreiteilung von Hochzeitskompositionen im Barock und sieht folgendermaßen aus:
A. Lob und Huldigung (Strophe 1-6)
B. Hinweise auf die Hochzeitsnacht (Strophe 7-8)
C. Wunsch nach Thronfolgern (Strophe 9)

In der Argumentation orientiert sich das Gliederungsschema der Komposition an Schemata, wie sie auch in Gelegenheitsdichtungen im Barock zu finden sind und beginnt wie viele andere Texte aus dieser Zeit mit der Allegorie von Lebensgenuss und Nichtigkeit menschlichen Daseins bzw. Vergänglichkeit im Gegensatz zum ewigen Leben. Doch danach wird es unterlassen Ulrika Eleonora oder Karl XI. zu preisen, sondern das Gefühl der Liebe verbunden mit Referenzen auf die bevorstehende Hochzeitsnacht und dem Einfordern von Nachkommenschaft bilden den Schwerpunkt. Letzteres unter kollektiver Zuwendung an das Brautpaar. Der Text der Komposition weist zwar die für den rituellen Kontext angemessen erscheinenden lyrischen Sprechakte auf wie: Anrede, Preisen, Rühmen und folgt somit einem bewährten Muster, aber es geht nicht um ein Rühmen und Preisen der Tugenden der Braut, sondern der Liebe. Auch die kollektive Adressierung von Braut und Bräutigam ist als normabweichend in diesem Kontext zu sehen. Diese zwei Besonderheiten des Textes (das Preisen des Liebesgefühls und die kollektive Adressierung) verbunden mit der Thematisierung von Sinnlichem evozieren ein Bild der zwischenmenschlichen Beziehung nach dem Muster einer romantischen Paarliebe. Im Text wird das Gefühl der Liebe nicht nur in Aussicht gestellt, sondern ein sich schon innig liebendes Königspaar inszeniert.

\subsection{Thematisierung von Emotionen}

Als multikodaler Text verstanden ist Dübens Komposition durch vielfältige Referenzen angereichert. Gemeint sind hier Bezüge nicht nur zu anderen semiotischen Zeichen, sondern auch zu emotiven und kognitiven Wissensbeständen. Im letzteren Fall stellt der Text Bezüge zum aktuellen Geschehnis der Hochzeit und ih- 
rer gesellschaftlich-politischen Verankerung her und diese Verankerung wird zwar zum kommunikativen Gerüst gemacht, doch die Kodierungen von Emotionen füllen dieses Gerüst mit Sinn und Inhalt. Die Kodierungen in Form von Emotionsthematisierungen, die an emotionsausdrückenden Verbalisierungen gekoppelt sind wie Emotionslexik, Metaphern und Exklamativsätzen kreieren zusammen mit Emotionspräsentationen wie Topoi und Emblematik Szenarien, in denen ein emotionales Erleben eingebettet wird. Gleichzeitig werden in diesen Szenarien die Bedingungen angeben, unter denen eine Emotion aktuell wird. Der Text von Alles Leben dieser Erden bildet diesbezüglich keine Ausnahme und umfasst somit Schablonen über emotionale Erfahrungen und über die Gestalt von Emotionen. Dadurch wird ebenfalls vorgeprägt, welche Praktiken und Ausdrucksformen im Zusammenhang mit Liebe angemessen und verständlich erscheinen oder zumindest noch zu rechtfertigen sind.

Dass während Zeremonien und Aufführungen von Gelegenheitskompositionen Gefühle hervorgerufen werden sollten, erscheint somit genauso nachvollziehbar wie die Benutzung von emotionsthematisierenden Bezeichnungen, die an emotionsausdrückende Verbalisierungen gekoppelt sind. Diese emotionsausdrückenden Verbalisierungen benennen die Gefühle nicht kategoriell, sondern bringen sie über die Semantik ausgewählter Lexeme zum Ausdruck: bestehn, treulich, suss, schön, wohlgefält. Dabei wird auch die stark positive Bewertung des Gefühls der Liebe ersichtlich, was eine Voraussetzung für eine stabile Tradierung des Liebes-Konzeptes über die Jahrhunderte hinweg bildet, was jedoch nicht ausschließt, dass dieses Konzept angereichert und verändert wird.

Im sprachlichen Kode sind aber auch Vorstellungen enthalten, wie Emotionen miteinander verbunden sind. Ein Gefühl kann als Vorbedingung für ein anderes aufgefasst werden:

Lieben ist [...] das Hertzens beste Ruht (Z. 5)

Lieben [...] gibt wergnugung, Ruh' wnd Fried (Z. 14)

Gefühle können auch im Verhältnis von Substituten oder antithetisch einander zugeordnet sein:

Lieben stillt des Neides Hassen (Z. 14)

Leben ohne Lieb’ wnd Lieben, bringet Kümmer wnd Betruben. (Z. 6)

Und obwohl negative Gefühle thematisiert werden (Kummer, Betrüben, Pein, Sorg, Leid, Neid, Hass) dienen diese als Kontrast, um die Kraft und Stärke der Liebe, ihre Vorherrschaft und Überlegenheit zu anderen Gefühlen zu inszenieren. So wird Liebe hierarchisch als das oberste Gefühl aller Gefühle dargestellt und das Lieben als die wichtigste christliche Praxis.

Einen bedeutenden Teil des Kodes bilden auch - über die Gefühls- und Ausdrucksregeln hinausgehend - die Vorstellungen über Steuerbarkeit und Kontrollierbarkeit von Gefühlen und Ausdruck:

Lieben muß den Stich behalten wnd mit keiner Zeit weralten (Z. 3)

Aller Menschen thun wnd Zielen zielt auf solche Zucker Lust [Liebe] (Z. 5) 
Ein starkes emotionales Wirkungspotential wird im Text durch die Verknüpfung von lexikalischen mit syntaktischen Mitteln erreicht. Neben dem Lexem Liebe und der Kollokation Liebe fühlen fallen die vielen Exklamativsätze auf, die Liebe als emotionalen Prozess darstellen und zur starken Emotionalität und Zügellosigkeit ermahnen sollen. Dies geschieht im Text entweder innerhalb einer Strophe (Nun so liebt Ihr Lieben Beijde, liebt Euch hertzlich fruh wnd Spat) oder die gesamte Strophe besteht aus Exklamativsätzen wie Strophe 9:

9. Euer Lieben laße schauen was der Liebe Lust wermag,

Eß wermehre sonder grauen Eure Lieb sich alle Tag;

Eß wermehre sich das Lieben, wnd was Euch hier zu getrieben.

Durchgängiges Prinzip der Textgestaltung ist die Wiederholung (eine Überspezifizierung durch Rekurrenz) sowohl des Lemmas lieb als auch der Exklamativsätze und auch der Metaphern. Betrachtet man die rhetorischen Figuren, in denen das Gefühl thematisiert wird, fallen folgende Muster auf:

a) Allegorie durch Personifizierung des Gefühls: Lieben herschet in dem Himmel, wnd in diesem Welt getummel

b) Antithetik: Lieben stillt des Neides Hassen

c) rhetorische Pathosformeln: Lieben ist das süsse spielen wnd das Hertzens beste Ruht

d) Alliteration: Leben ohne Lieb' wnd Lieben

e) Metapher: Lieben hat sein Hertz getrieben, daß Er nun mit liebes Flamm wnd mit tausend Zucker-Küssen wil sein Leben uber sussen

In der Komposition sind sowohl wesensbeschreibende metaphorische Konzepte (LIEBE IST ZUCKER) als auch handlungsbezogene metaphorische Konzepte (LIEBE IST SPIEL) vertreten. Als konzeptionell-metaphorische Profile dienen - in Lakoffs und Johnsons (1980) Terminologie - das BEHÄLTER-Konzept und das KRAFT-Konzept: Liebe ist Feuer. Hierbei ist LIEBE sowohl Behältersubstanz (Lieben ist [...] das Hertzens beste Ruht) als auch Behälterobjekt: Liebe ist der Hertzen Himmelreich. Durch Aufgreifen der Höhenmetapher und auch der Thematisierung der Liebesgöttin Venus (Eß beschütz fur Sorg wnd Leide Wenuß euch mit Rath wnd that) werden sowohl Religion als auch Mythologie in den Dienst des Gefühls gestellt. Die Komposition bedient sich somit einiger im Barock geläufiger Sinnbilder, die in ihrer soziopragmatischen Bedeutung weitgehend festgelegt sind.

Die im Text benutzten emotionsthematisierenden Mittel weichen somit nicht von gängigen und für Gelegenheitskompositionen etablierten ab. Erst bei der Betrachtung der Topoi an der Schnittstelle von Emotionsthematisierungen und Emotionspräsentationen lassen sich Besonderheiten feststellen. 


\subsection{Bevorzugte Topoi}

Als historisch manifeste Denkmodelle, die die Wirklichkeit ordnen, besitzen Topoi zumindest ein hypothetisches Erklärungspotential ${ }^{17}$. Die Anbindung der Analyse von Topoi an den kodebasierten Ansatz und somit an die Emotionspräsentation (im Zusammenwirken mit der Emotionsthematisierung) ermöglicht einzelne Bestandteile emotionaler Inszenierung und Kommunikation in den Blick zu bekommen und gleichzeitig Einsichten in ein kollektives, gesellschaftliches Wissen aus dem Text zu gewinnen. Die wiederholte Verschränkung von inhaltlichen und formalen Aspekten ist ein durchgängiges Prinzip bei den Topoi. So wird der zu erwartende Ewigkeitstopos der Liebe in der Komposition kumulativ durch Sentenzen gestaltet, in denen das Lemma lieb größtenteils immer präsent ist.

(1) lieben bleibet fest bestehn (Z. 2)

(2) Lieben muß den Stich behalten wnd mit keiner Zeit weralten (Z. 3)

(3) Lieben ist das süsse spielen wnd das Hertzens beste Ruht (Z. 4)

(4) Leben ohne Lieb' wnd Lieben, bringet Kümmer wnd Betruben (Z. 6)

(5) Lieben wnd geliebet werden ist das beste Spiel auff Erden (Z. 9)

(6) Durch dir liebe wird den wunden der werliebten Raht gefunden (Z. 12)

(7) Lieben stillt des Neides Hassen, gibt wergnugung, Ruh' wnd Fried (Z. 14)

(8) Lieben kan durch susses lachen bittre Gall zu Honig machen (Z. 15)

(9) das werliebte Lieben ist das leben dieser Welt (Z. 16)

Die zeitlose Präsenz des Gefühls Liebe wird durch diesen Topos sowohl inhaltlich-thematisch mit: Liebe ist/kan/muss/gibt... usw. getragen, als auch mit sprachlicher Formelhaftigkeit wie mit Hilfe von Alliteration (Leben ohne Lieb' wnd Lieben), Paarformeln (Rath wnd that, Ruh' wnd Fried, fruh wnd Spat) und Redensarten (Leben ohne Lieb' wnd Lieben, bringet Kümmer wnd Betruben).

Diese Gelegenheitskomposition vermittelt inhaltlich-thematisch aber nicht nur ein Bild über die Ewigkeit des Gefühls, sondern erstaunt durch das Ausblenden von gesellschaftlichen Moralvorstellungen wie Treue, Frömmigkeit, Demut und Umsichtigkeit (vgl. Snickare 1999, 24). Aber auch für das 17. Jahrhundert gängige Tugenden wie Mitleid, Aufrichtigkeit und Ehrlichkeit, Geduld, Wachsamkeit und Vorsicht werden hier nicht aufgegriffen.

Statt einer moralisierenden Narration wird im Kontext des Gefühls die Hingabe emphasiert. Die dafür benutzten Topoi sind der Vergnügungstopos (Z. 4, 9, 11, 14,

\footnotetext{
17 Der Begriff »Topos« wird in der aristotelischen Rhetorik (s. in Rapp/Corcilius 2011, S. 511) sowohl als Suchformel für Argumente als auch als Beweisformel verstanden. In der Funktion als Suchformeln sind die Topoi als »Wohnsitze für Argumente« definiert worden und in der Funktion als Beweisformel als Garantie für die Relevanz von Argumenten gesehen worden. Für die Gelegenheitskompositionen ist mit Topoi in Anlehnung an Wengeler (2007, S. 165 ff.) ein argumentatives Gewohnheitswissen gemeint, dass durch die in sprachlichen Handlungen wiederholte Verschränkung von inhaltlichen und formalen Aspekten Einsichten in ein kollektives, gesellschaftliches Wissen vermittelt. Mit Topoi kann einerseits der habitualisierte Gebrauch gleicher Schlussmuster als ein Bestandteil sozialen Wissens erforscht, andererseits können sie auch aufgefasst werden als Bestandteil des jeweils gerade gültigen sozialen Wissens zu einem Thema. Eine forschungsgeschichtliche Übersicht zum Topos-Begriff und seiner Tradierung in der Linguistik ist auch bei Wengeler (2007) einsehbar.
} 


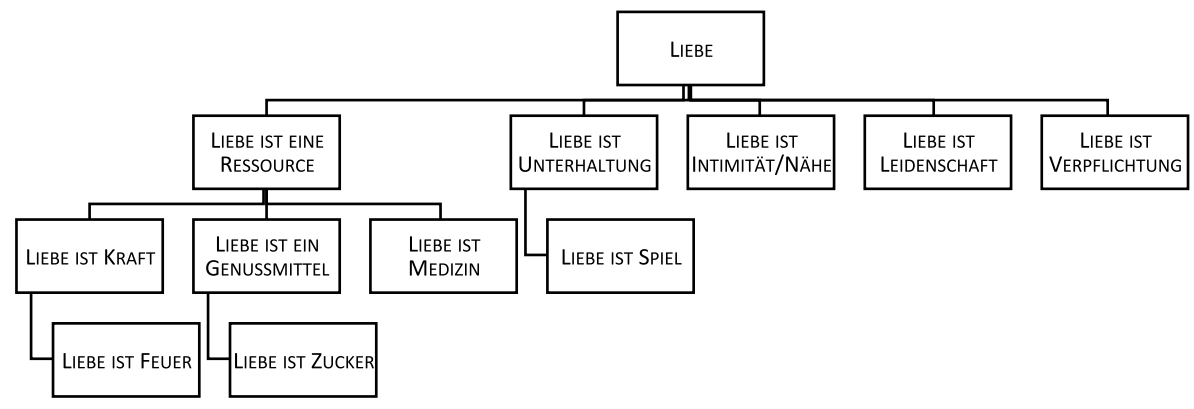

Abb. 4 Schematische Darstellung des Gefühlskonzepts LIEBE in Alles Leben dieser Erden (1680)

\section{8, 25) der Topos des Genusses (Z. 5, 7, 11, 21) und der Topos der Leidenschaft} (Z.16-17, 19-21, 22, 26), die eindeutig in der Komposition dominieren.

Insbesondere die Inhalte in den Strophen 7 bis 9 sind gewagt gestaltet - im Imperativ gehalten findet sich hier die deutliche Aufforderung zur körperlichen Liebe und Lust: Nun so liebt Ihr Lieben Beijde, liebt Euch hertzlich fruh wnd Spat (Z. 22); E $\beta$ wermehre sich das Lieben, wnd was Euch hier zu getrieben (Z. 27).

Obwohl die zu erwartende Mutterrolle ${ }^{18}$, den Thronfolger zu gebären und somit die Dynastie zu sichern und Kontinuität zu gewähren, nicht explizit auf der Textoberfläche sichtbar wird, wird diese Rollenerwartung eingefordert und ist metaphorisch in Strophe 9 vorhanden. Hier wird Liebe als Verpflichtung zum Erzeugen von Nachwuchs thematisiert (Euer Lieben laße schauen was der Liebe Lust wermag) und als Ursache für Kindersegen (Strophe 9):

$[\ldots]$

Eß wermehre sonder grauen Eure Lieb sich alle Tag;

Eß wermehre sich das Lieben, wnd was Euch hier zu getrieben.

Über die metaphorische Gestaltung des Konzeptes Liebe und die Muster in der Thematisierung und Präsentation von Gefühlen in der Gelegenheitskomposition lässt sich ein schematischer Überblick erstellen (s. Abb. 4). Ersichtlich wird, dass sich schon hier Anzeichen eines sich zeitgeschichtlich später etablierten Muster der romantischen Paarliebe abzeichnen. So auch bei dem Konzept LIEBE IST VERPFLICHTUNG, wo dem Paar die Verantwortung für ein Leben in Liebe übertragen wird und demzufolge dieses nicht in Gottes Hand liegt.

Dass die Eheschließung einen Bestandteil des Friedensvertrags ausmacht, wird im Werk nicht besungen. ${ }^{19}$ Die Korrelation zwischen Frieden und Eintracht einerseits und Liebe andererseits wird somit nicht in aller Deutlichkeit thematisiert. Das Gefühl wird einzig und allein in den unterschiedlichsten sprachlichen Ausformungen vorgestellt, was entweder damit zusammenhängen mag, dass der Text der Hochzeits-

\footnotetext{
18 Außer ihrer Mutterrolle sollte sie in den Jahren als Königin in Schweden die Rolle als »Mutter des Volkes « übernehmen (Snickare 1999, S. 73).

19 Man könnte aber die Zeilen in Strophe 5 »Lieben stillt des Neides Hassen, gibt wergnugung, Ruh' wnd Fried « (Z. 14) und »Lieben kan durch susses lachen bittre Gall zu Honig machen« (Z. 15) als Anspielung auf die kriegerische Vorgeschichte deuten.
} 
musik zeremoniellen Regeln verpflichtet ist oder auch Umstände spiegelt, die insbesondere für diese königliche Eheschließung vorlagen und zwar die reelle Existenz von irgendeiner Form von Gefühlen ${ }^{20}$ wie Zuneigung, Verbundenheit, Zusammengehörigkeit, die textgattungsspezifisch und im Kontext der Zeremonie als positiv ausgedeutet wurden und auszudeuten sind.

\section{Ausblick}

Die Arbeit mit Gelegenheitskompositionen zeigte deutlich, dass Texte aus dem 17. Jahrhundert ohne eine Kontextualisierung weder für die Menschen, die mit ihnen lebten, noch für uns, die den Sinn irgendwie rekonstruieren wollen, leicht $\mathrm{zu}$ erschließen sind. Aus einer theoretisch-methodischen Perspektive impliziert dies, dass kulturanalytisch-linguistisches Arbeiten mit Texten stets ein $(\mathrm{Re})$ Kontextualisierungsverfahren verlangt, welches ermöglichen soll, diese nicht losgelöst von dem Entstehungs- und Rezeptionsprozess und ihrem Wirkungsraum $\mathrm{zu}$ sichten.

Die historisch interessante Gelegenheitskomposition von Gustav Düben Alles Leben dieser Erden (1680) im kulturellen Entstehungs- und Aufführungszusammenhang zu betrachten, mit Fokus auf die rollenspezifischen Erwartungen an die Königin Ulrika Eleonora, bedeutet somit einerseits, dieses Werk in zeremoniellen Zusammenhängen zu sehen, und andererseits Komponenten für die Sinngebung von Zeremonien zu erschließen, denn der Sinn der Komposition wird vorrangig durch die Einbettung in die Zeremonie geschaffen. Beteiligt an der Sinngebung sind Text und Musik, die sich in ihrer Darstellungs- und Ausdruckskraft ergänzen. Unumgänglich ist aber nicht nur die Funktion der Gelegenheitskompositionen dem feierlichen Anlass eine entsprechende Umrahmung zu verleihen, sondern auch bestimmte Affekte bei den Rezipienten in dem sozialen Geschehen des Festes zu erwecken.

Bei aller Nähe der beiden Zeichensysteme Text und Musik werden Transkriptivitätseffekte erzeugt, also Effekte, die entstehen, wenn man den Text zur Explikation der Musik heranzieht, um das gesamte ästhetische Produkt lesbar zu machen, d.h. seine Bedeutung zu erschließen oder überhaupt erst zu konstituieren, denn auf Grund ihrer Semantik verfügen Texte über ein Referenzpotential, mit dem sie auf außersprachliche Sachverhalte Bezug nehmen können. So kann die Komposition dazu beitragen, den bestimmten Anlass zur Feier, die Adressaten und schließlich auch den Autor zeitweise in den Vordergrund zu heben. Geleistet wird dies durch Emotionalisierungstechniken, die in der sprachlichen Ausgestaltung u.a. unter Verwendung besonderer emotionsthematisierender und -präsentierender Mittel und Topoi verankert liegen. Indem dies geschieht, konserviert die Komposition, als semiotischer Komplex von Text und Musik betrachtet, die Feierlichkeit und sich selbst als ästhetisches Produkt der Kultur des 17. Jahrhunderts.

\footnotetext{
20 Die Annahme von einem Vorhandensein von Gefühlen zwischen Karl XI. und Ulrika Eleonora ist nicht allzu abwegig, wenn man bedenkt, dass das Paar vier Jahre verlobt blieb, während der Schwedisch-Dänische Krieg wütete.
} 
Die Entstehung von Gelegenheitskompositionen am schwedischen Hof und im Auftrag des Königs ist immer im Kontext eines Anlasses zu sehen. Gustav Dübens Alles Leben dieser Erden ist nachweislich mit einer königlichen Hochzeitsfeier und somit einer Zeremonie verbunden. Auch wenn die Aufführung der Komposition dem Huldigungszweck dient und gesellschaftlichen Erwartungshaltungen explizit zum Ausdruck bringen kann, ist sie durch den Text eindeutig mit einem auffälligen Emotionspotential versehen, indem sie das Liebesgefühl thematisiert und präsentiert. In einem zeremoniellen Zusammenhang wie die Hochzeit des Regenten, die im 17. Jahrhundert ein politisches Ereignis darstellt, wird somit in dieser Komposition das Gefühl der Liebe ins Zentrum gesetzt und gehuldigt.

In ihrer Gesamtheit weist die Komposition Merkmale auf, die als zeittypisch und usuell für Kompositionen des Barock zu sehen sind. Sie folgt jedoch in einigen Einzelaspekten nicht den gängigen Vorlagen. Es fehlt in diesem Zusammenhang der Frömmigkeitstopos. Die Gelegenheitskomposition umreißt (im Kontext der Hochzeit) dagegen ein Bild der Liebe und darüber, wie und was das schwedische Königspaar gemeinsam in dieser Situation zu fühlen hat. Es wird ermahnt, zusammen Eigenverantwortung für das Liebesglück zu übernehmen und für einen Thronfolger zu sorgen und somit die Monarchie zu sichern. Präsentiert wird die Erwartungshaltung deutlich im Topos des Genusses, der Unterhaltung und der Leidenschaft.

Das Gefühl der Liebe wird als Gefühl gezeichnet, in dem eine bestimmte soziale Schicht im 17. Jahrhundert handeln und die Welt sehen soll. Appelliert wird an den homo sentiens. Es ist zu ersehen, dass Gelegenheitskompositionen nicht nur zufällige Thematisierungen von Gefühlen enthalten, sondern jeweils konstitutive Momente in der Thematisierungs-, Präsentations- und Wandlungsgeschichte von Gefühlen in jeweiligen gesellschaftlichen Kontexten spiegeln. Auch wenn im Zeitalter des Barock Gefühle stark emphasiert werden, so kann für die Hochzeitskomposition als Bestandteil einer Zeremonie festgehalten werden, dass hier nicht nur eine Darstellung des Liebesgefühls auf dem Hintergrund anderer Gefühle stattfindet, sondern dass dies gleichzeitig auf Kosten des Mäßigungstopos geschieht; auch andere zu erwartende Topoi werden übergangen. Als Motto gilt: Liebe vor Gottesfurcht und Frömmigkeit.

Open Access Dieser Artikel wird unter der Creative Commons Namensnennung 4.0 International Lizenz (http://creativecommons.org/licenses/by/4.0/deed.de) veröffentlicht, welche die Nutzung, Vervielfältigung, Bearbeitung, Verbreitung und Wiedergabe in jeglichem Medium und Format erlaubt, sofern Sie den/die ursprünglichen Autor(en) und die Quelle ordnungsgemäß nennen, einen Link zur Creative Commons Lizenz beifügen und angeben, ob Änderungen vorgenommen wurden.

\section{Quellen- und Literaturverzeichnis}

\section{Quellen}

Buxtehude, Dietrich: Klinget für Freuden (https://www2.musik.uu.se/duben/presentationWork.php? Select_Dnr=332\&Select_Wnr=252, ges. 6.12.2018)

Düben, Gustav: Alles Leben dieser Erden (https://www2.musik.uu.se/duben/presentationSource1.php? Select_Dnr=689, ges. 6.12.2018) 
Düben, Gustav: War wälkommen min bästa Wänn (https://www2.musik.uu.se/duben/presentationWork. php?Select_Dnr=707\&Select_Wnr=532, ges. 6.12.2018)

Geist, Christian: Beati omnes qui timent Dominum (https://www2.musik.uu.se/duben/presentationWork. php?Select_Dnr=883\&Select_Wnr=655, ges. 6.12.2018)

Geist, Christian: Sij huru godt och lustight är thet (https://www2.musik.uu.se/duben/presentationWork. php?Select_Dnr=2430\&Select_Wnr=698, ges. 6.12.2018)

\section{Literatur}

Berglund, Lars: Studier i Christian Geists vokalmusik. Diss. Uppsala 2002.

Braungart, Wolfgang: Ritual und Literatur. Tübingen 1996.

Burckhardt, Jacob: Die Cultur der Renaissance in Italien. Ein Versuch. Basel 1860.

Burke, Peter: Ludwig XIV. Die Inszenierung des Sonnenkönigs. 3. Auflage. Berlin 2009.

Ekman, Paul/von Friesen, Wallace V.: Unmasking the Face. A Guide to Recognizing Emotions from Facial Expressions. Englewood Cliffs 1975.

Erben, Dietrich: Paris und Rom. Die staatlich gelenkten Kunstbeziehungen unter Ludwig XIV. Berlin 2004.

Fischer, Peter-Michael: Die Stimme des Sängers. Analyse ihrer Funktion und Leistung - Geschichte und Methodik der Stimmbildung. 2. durchgesehene Auflage. Stuttgart/Weimar 1998.

Fix, Ulla: »Text und Textlinguistik«. In: Nina Janich (Hg.): Textlinguistik. 15 Einführungen. Tübingen 2008, S. 15-34.

Geertz, Clifford: Dichte Beschreibung. Beiträge zum Verstehen kultureller Systeme. Frankfurt a.M. 1991.

Harré, Rom: The Social Construction of Emotions. Oxford 1986.

Herzog, Urs: Deutsche Barocklyrik. Eine Einführung. München 1979.

Hillebrandt, Claudia: Das emotionale Wirkungspotenzial von Erzähltexten: Mit Fallstudien zu Kafka, Perutz und Werfel. Berlin 2011.

Jahr, Silke: Emotionen und Emotionsstrukturen in Sachtexten: ein interdisziplinärer Ansatz zur qualitativen und quantitativen Beschreibung der Emotionalität von Texten. Berlin/New York 2000.

Lakoff, George/Johnson, Mark: Metaphors We Live By. Chicago/London 1980.

Lenz, Karl: Soziologie der Zweierbeziehung. Eine Einführung. 2. aktualisierte und überarbeitete Auflage. Wiesbaden 2003.

Mattheson, Johann: Der Vollkommene Capellmeister. Hamburg 1739.

Molière: »Air de Ballet De M. Batiste «. In: Alphonse Lemerre (Hg.): Poésies diverses attribuées à Molière ou pouvant lui être attribuées. Paris 1869, S. 112.

Myerhoff, Barbara: Number Our Days. New York 1978.

Norlind, Tobias: Die Musikgeschichte Schwedens in den Jahren 1630-1730. Leipzig 1900.

Rapp, Christof/Corcilius, Klaus: Aristoteles-Handbuch: Leben - Werk - Wirkung. Stuttgart/Weimar 2011.

Saralja, Jukka: Music, Morals, and the Body. An Academic Issue in Turku 1653-1808. Helsinki 2001.

Schildt, Maria: Hyllningsmusik till Karl XI - en studie kring 18 tillfälleskompositioner. Uppsala 2007.

Schildt, Maria: Gustav Düben at Work. Musical repertory and practice of Swedish court musicians, 1663-1690. Diss. Uppsala 2014.

Schwarz-Friesel, Monika: Sprache und Emotion. Tübingen/Basel 2007.

Schwarz-Friesel, Monika: »Das Emotionspotenzial literarischer Texte«. In: Anne Betten/Ulla Fix/Berbeli Wanning (Hg.): Handbuch Sprache in der Literatur. Berlin/Boston 2017, S. 351-370.

Snickare, Mårten: Enväldets Riter. Kungliga Fester och Ceremonier i Gestaltning av Nicodemus Tessin den Yngre. Stockholm 1999.

Stoeva-Holm, Dessislava: »Gefühle worten. Zum Emotionalisieren in zeitgenössischer Literaturkritik«. In: Heinrich Kaulen/Christina Gansel (Hg.): Literaturkritik heute: Tendenzen - Traditionen - Vermittlung. Göttingen 2015, S. 27-42.

Strong, Roy: Art and power: Renaissance festivals 1450-1650. Woodbridge 1973.

Turner, Victor W.: The Anthropology of Performance. New York 1988.

Vowinckel, Gerhard: »Zivilisationsformen der Affekte und ihres körperlichen Ausdrucks«. In: Zeitschrift für Soziologie, 18, Heft 5, 1989. S. 362-377.

Wengeler, Martin: »Topos und Diskurs - Möglichkeiten und Grenzen der topologischen Analyse gesellschaftlicher Debatten«. In: Ingo H. Warnke (Hg.): Diskurslinguistik nach Foucault. Berlin 2007, S. 165-186.

Winko, Simone: Kodierte Gefühle. Zu einer Poetik der Emotionen in lyrischen und poetologischen Texten um 1900. Berlin 2003. 